\title{
Analysis of user behaviour at a roundabout under car-following conditions
}

\author{
G. Guido \& A. Vitale \\ Department of Town and Country Planning, University of Calabria, Italy
}

\begin{abstract}
Roundabout intersection planning in urban areas has spread remarkably, especially in Europe. Compared with the conventional intersection layout, this system is suitable for improving road safety and the urban landscape. In the literature there are many studies regarding car-following models; the innovative characteristic of this research is the analysis of user behaviour at a roundabout. In fact, the main aim is to develop a car-following model allowing the definition of flow conditions under high vehicular densities at this type of intersection. The car-following model consists of a stimulus-response function; its calibration and validation has been made by the analysis of many parameters influencing traffic flow. These parameters have been observed with a video data acquirement technique in order to minimize the distance between the observed variables and the estimated one. Preliminary results show a strong correlation between the observed behavioural parameters and the output of the model, forecasting vehicular trajectories at a roundabout. The estimation of traffic parameters in car-following condition is useful to define the geometry of the intersection (length of weaving area, number of lanes, diameter, number of entries) and to forecast conditions that could lead to behavioural anomalies connected with a reduced safety level for users.
\end{abstract}

Keywords: driver behaviour, roundabout, car-following, model.

\section{Introduction}

The conditions of circulation on the road network have never been more important, above all in the urban context. In fact, vehicular traffic congestion produces increasingly frequent inconvenience to the users and causes negative effects in terms of safety and environmental pollution. 
The solution to these problems should not be sought in the realisation of new infrastructures, but rather in the shrewd management of mobility and in accurate transport planning. The study of user behaviour on the road system is one possible means of support to the planning and management of the system. It is into this context that the research presented in this paper is presented. In this work, the authors, who previously drew attention to the behavioural model of lane change on roundabouts [1], focus on the observation of the phenomenon of car-following on urban roundabouts. The originality of the work lies in the fact that, contrary to the models available in the literature, an analysis is made of user behaviour in car-following conditions on curvilinear trajectories (on the roundabout) and with a very different profile from those encountered in rectilinear circumstances.

\section{Literature review}

The origin of the studies carried out on user behaviour in conditions of car-following dates from midway through the last century. At that time, Chandler et al. [2] formulated a first car-following model, based on the theory that user acceleration, in the shape of a stimulus-response type, was proportional to the difference in speed among themselves and the preceding vehicles in the same lane.

$$
a_{n}(t)=\alpha \cdot \Delta V_{n}^{\text {front }}\left(t-\tau_{n}\right)
$$

where, $\tau_{\mathrm{n}}$ is the perception time of the driver $n ; \mathrm{a}_{\mathrm{n}}(\mathrm{t})$ the (response) acceleration applied by the driver $n$ at time $t ; \Delta \mathrm{V}_{\mathrm{n}}^{\text {front }}\left(\mathrm{t}-\tau_{\mathrm{n}}\right)$ the (stimulus) speed difference between user $n$ and the preceding vehicles in the same lane at time $t$ - $\tau_{n}$; and $\alpha$ is a (sensitivity) constant.

On the basis of this first formulation, Gazis et al. [3] suggested a model that took into account the space headway between two successive vehicles in terms of sensitivity, whose analytical definition is represented by the following:

$$
a_{n}(t)=\frac{\alpha}{\Delta X_{n}\left(t-\tau_{n}\right)} \cdot \Delta V_{n}^{\text {front }}\left(t-\tau_{n}\right)
$$

where, $\Delta \mathrm{X}_{\mathrm{n}}\left(\mathrm{t}-\tau_{\mathrm{n}}\right)$ is the space headway between user $n$ and the preceding vehicles in the same lane at time $t-\tau_{n}$.

Many calibrations of the model were carried out from 1959 onwards [4, 5] using various approaches and on different scales (microscopic and macroscopic).

The more general formulation of the model, proposed by Gazis et al. [6], known as the General Motors Nonlinear Model, considers the term of sensitivity directly proportional to the speed $\left(\mathrm{V}_{\mathrm{n}}(\mathrm{t})\right)$, elevated to a coefficient $(\beta)$, and inversely proportional to the space headway $\left(\Delta \mathrm{X}_{\mathrm{n}}\left(\mathrm{t}-\tau_{\mathrm{n}}\right)\right)$, elevated to a coefficient

$(\gamma)$; the stimulus is represented by the difference of speed $\left(\Delta \mathrm{V}_{\mathrm{n}}{ }^{\text {front }}\left(\mathrm{t}-\tau_{\mathrm{n}}\right)\right)$ among the successive vehicles. 


$$
a_{n}(t)=\alpha \frac{V_{n}\left(t-\tau_{n}\right)^{\beta}}{\Delta X_{n}\left(t-\tau_{n}\right)^{\gamma}} \cdot \Delta V_{n}^{\text {front }}\left(t-\tau_{n}\right)
$$

where, $\mathrm{V}_{\mathrm{n}}(\mathrm{t})$ speed of the driver $n$ at time $t-\tau_{n}$.

Therefore, the formulations defined by Chandler et al. [2] and by Gazis et al. [3] can be considered special cases derived by the above-mentioned model.

The development of technologies available to record vehicle flow parameters enables the definition of more general models and the estimation of the best combination of constants $\beta$ and $\gamma,[7-9]$.

In the last twenty years, new calibrations of the model have been carried out considering different analysis phases, that is, in deceleration, acceleration and steady state conditions [10].

In 1996, Subramanian [11] proposed an analogous formulation to that of the GMN model, in which a random term appears associated with the user $\mathrm{n}$ at time $\mathrm{t}$ distributed normal and in which perception and reaction time $\tau$ is considered as a random variable distributed truncated lognormal:

$$
a_{n}(t)=\alpha \frac{V_{n}\left(t-\tau_{n}\right)^{\beta}}{\Delta X_{n}\left(t-\tau_{n}\right)^{\gamma}} \cdot \Delta V_{n}^{f r o n t}\left(t-\tau_{n}\right)+\varepsilon_{n}(t)
$$

In 1999, Ahmed [12] formulated a new expression of the GMN model, considering the effect of vehicle density $\left(\mathrm{K}_{\mathrm{n}}\left(\mathrm{t}-\varepsilon \tau_{\mathrm{n}}\right)\right)$ in the functioning of sensitivity and suggesting a non-linear relation between acceleration and the stimulus function $\left(\Delta \mathrm{V}_{\mathrm{n}}{ }^{\text {front }}\left(\mathrm{t}-\varepsilon \tau_{\mathrm{n}}\right)\right)$ :

$$
a_{n}(t)=\alpha \frac{V_{n}\left(t-\varepsilon \tau_{n}\right)^{\beta}}{\Delta X_{n}\left(t-\varepsilon \tau_{n}\right)^{\gamma}} \cdot K_{n}\left(t-\varepsilon \tau_{n}\right)^{\rho} \cdot\left|\Delta V_{n}^{\text {front }}\left(t-\varepsilon \tau_{n}\right)\right|^{\lambda}
$$

in which $\varepsilon(\in(0 ; 1))$ is an adaptation parameter of the perception of the congestion level by user $n$.

The variability of the estimate of the model coefficient values, found from an analysis of the calibrations and from the validations of the GMN model proposed by various researchers, suggests some considerations. The vehicle flow conditions, differing in the various experimental contexts, considerably influence the model parameters. Consider the weight, moreover, the level of analytical approach (macro-simulation and micro-simulation) has on the calibration of the model, the phases of motion analysis (acceleration, deceleration and steady state) and the different assumptions made on the distribution of the $\tau$ values.

Besides the previously-described models, based on the stimulus-response function, car-following models based on the concept of braking distance have been developed, [13-15], as well as models based on linear relations, [10, 16-19], psychophysical models, [20-23], models based on fuzzy logic, [24-27], and other models based on innovative techniques, [28-30]. 


\section{Calibration of the General Motors Nonlinear Model on the roundabout situated near the Cosenza North motorway turnoff}

From an analysis of the car-following models in the literature, the need arose to calibrate the most general model (GMN model), revised by Subramanian [11], for circulation conditions substantially different from those found in the previous experiments. Therefore, vehicle flow was analysed at a node of the road network of a medium-sized town in southern Italy (Cosenza) and, specifically, at an intersection with a roundabout (figure 1).

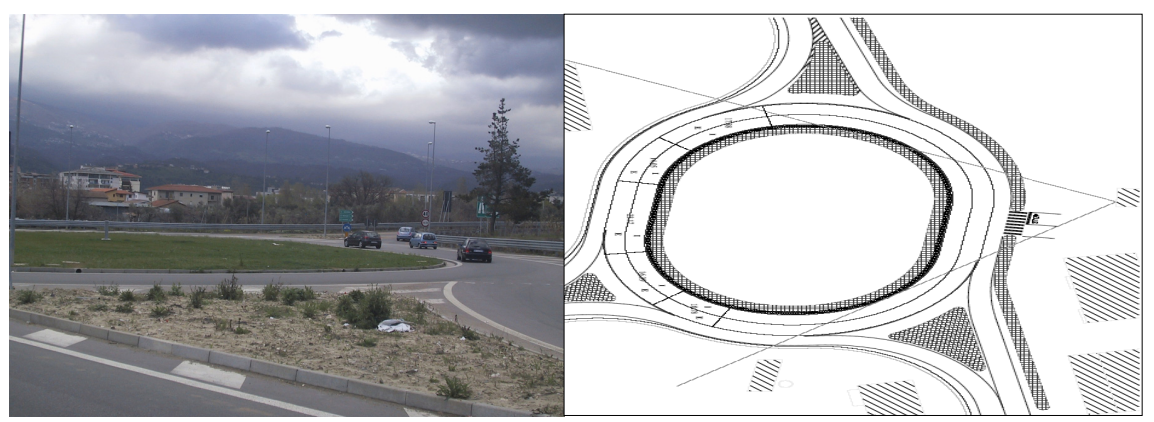

Figure 1: $\quad$ Surveyed roundabout.

\subsection{Data gathering}

The area under examination is close to the Cosenza North motorway turnoff, which is an important node in the local road network owing to the presence, in the neighbourhood, of the most important regional university complex. Considerable vehicle flows use this node and a study was required with the aim of improving the outflow conditions.

The geometry and parameters characterising the vehicle flow on the roundabout were recorded. The database was constructed by means of a video data acquirement process.

First, the a O/D matrix was analysed at rush hour, individuating the busiest entries of the roundabout and recording the circulatory flow on the roundabout itself.

It emerged from the data analysis that car-following conditions occur, in accordance with Aycin and Benekohal's theory [31], for distances of less than 75 metres: in fact, for distances greater than that critical value, user behaviour does not prove to be in any way influenced by preceding vehicles in the same lane.

With the aim of determining parameters useful for the definition of the car-following model, the area monitored was discretized into further trunks. This 
discretization was carried out with the purpose of finding the instantaneous characteristics of the motion of each vehicle.

The quantities found are the following:

$V_{n}(t)$ the speed of the user $n$ (subject) at instant $t$;

$\mathrm{V}_{\mathrm{n}}\left(\mathrm{t}-\tau_{\mathrm{n}}\right)$ the speed of the user $\mathrm{n}$ (subject) at instant $\mathrm{t}-\tau_{n}$;

$\mathrm{V}_{\mathrm{n}-1}\left(\mathrm{t}-\tau_{\mathrm{n}}\right)$ the speed of the user's predecessor at instant $\mathrm{t}-\tau_{n}$.

It should be pointed out that the determination of the perception and reaction time $(\tau)$ of the user derives from the experimental relation contained in the Ministerial Decree 05/11/2001 (Functional and geometrical norms for road building), in which:

$$
\tau_{n}=2.8-0.01 \cdot V_{n}(t)
$$

The determination of the acceleration applied by the user $n$ (subject) at instant $t$ was carried out with the following equation:

$$
a_{n}(t)=\frac{V_{n}(t)-V_{n}\left(t-\tau_{n}\right)}{\tau_{n}}
$$

\subsection{Data elaboration}

From analysis of the sample, it emerges that a predominant percentage of the manoeuvres were carried out in deceleration conditions; for this reason, the authors calibrated a general deceleration model in car-following conditions.

Prior to calibration of the model, a statistical analysis was carried out to determine the correlation among the observed quantities.

The deceleration applied by the $n$-th user at instant $t$ proved to vary linearly both with velocity $\mathrm{V}_{\mathrm{n}}$ and with $\Delta \mathrm{V}_{\mathrm{n}}$, whereas the relation between the same quantity $a_{n}$ and the spatial distancing $\Delta X_{n}$ proved to be of an exponential type (figures 2, 3 and 4).

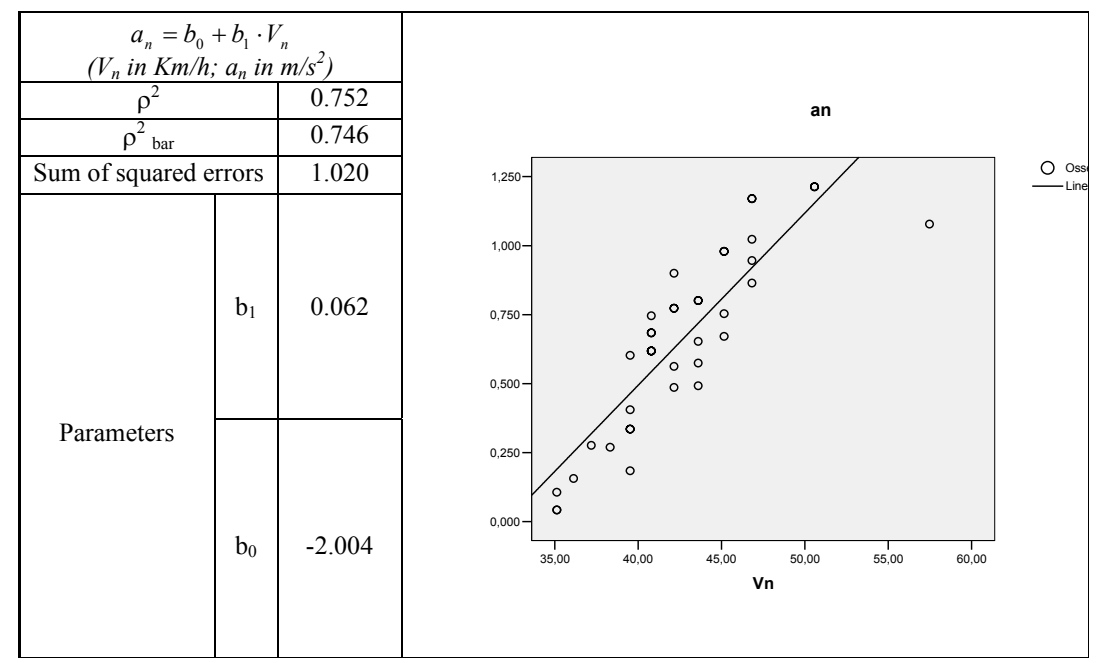

Figure 2: $\quad$ Correlation between $a_{n}-V_{n}$. 


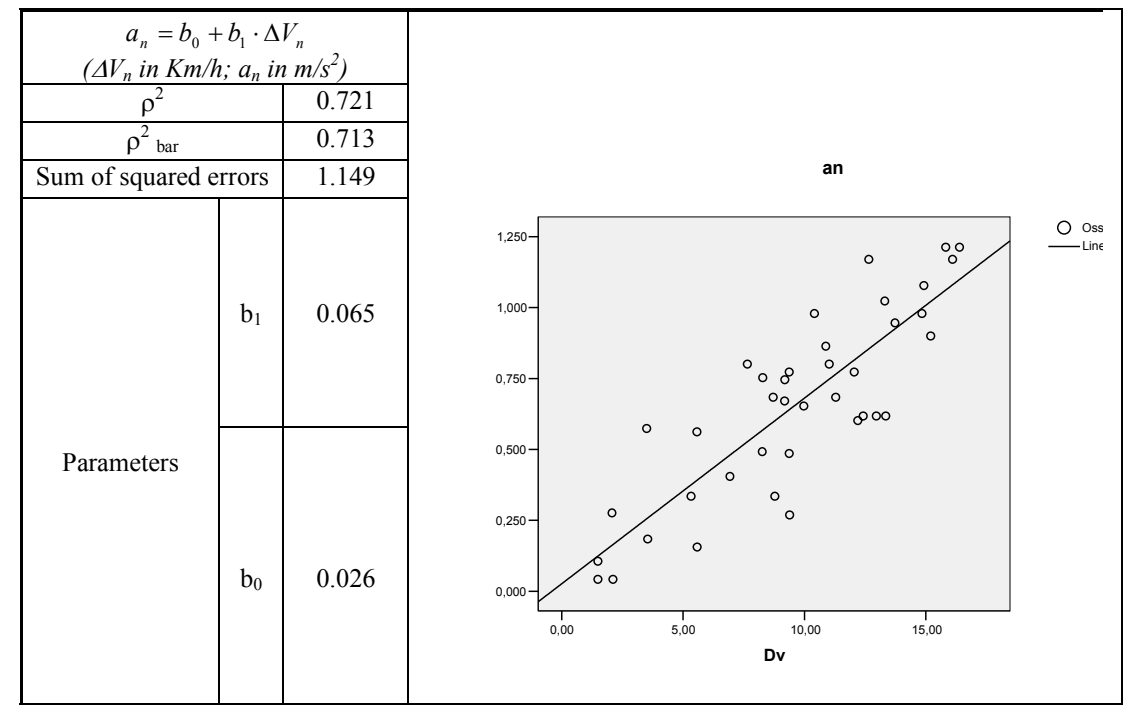

Figure 3: Correlation between $a_{n}-\Delta V_{n}$

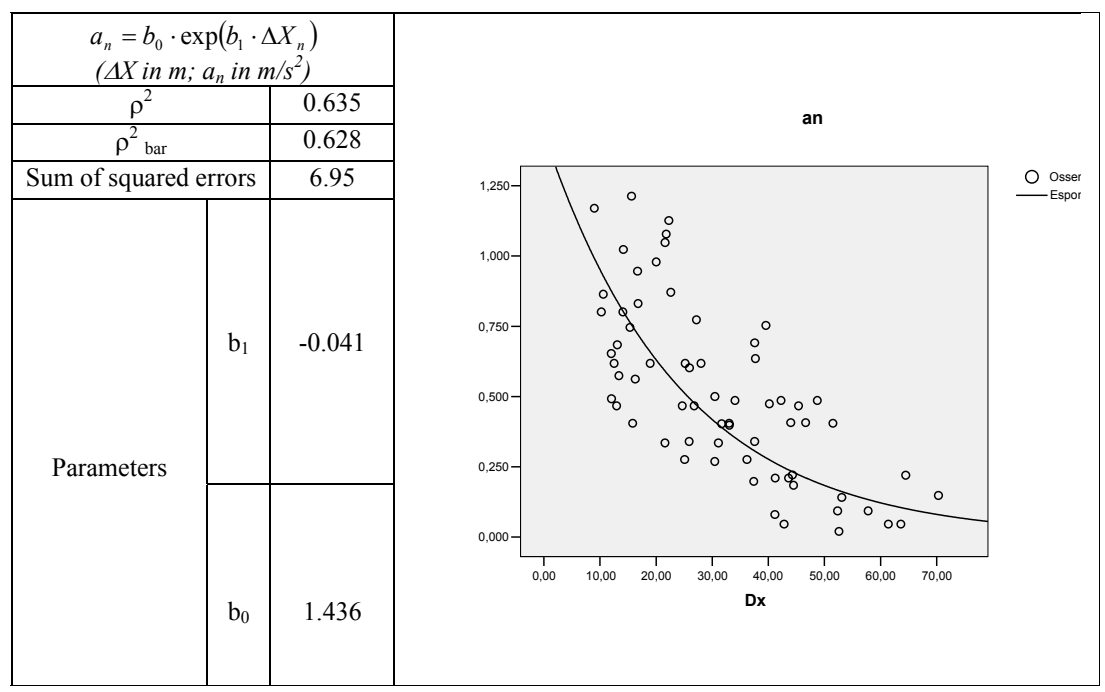

Figure 4: Correlation between $a_{n}-\Delta X_{n}$.

\subsection{Calibration of the model}

The estimate of the parameters of the model $(\alpha, \beta, \gamma)$ was carried out using the nonlinear least square method (Nonlinear Least Square Analysis). In this case, the function to be minimised is not quadratic in relation to the unknown parameters, consequently the solutions were sought by means of the LevenbergMarquardt algorithm. The results of the estimate are shown below (table 1). 
Table 1: $\quad$ Model calibration results.

\begin{tabular}{|l|c|c|c|c|}
\hline & \multicolumn{2}{|c|}{ Sum of squares } & \multicolumn{2}{c|}{ Mean squares } \\
\hline Regress. & \multicolumn{2}{|c|}{50.685} & \multicolumn{2}{c|}{16.895} \\
\hline Res. & \multicolumn{2}{|c|}{6.021} & \multicolumn{2}{c|}{0.060} \\
\hline$\rho_{\text {bar }}^{2}$ & \multicolumn{2}{|c|}{0.679} & \multicolumn{2}{|c|}{ Fiducial limits } \\
\hline \multirow{2}{*}{ Parameters } & Estimate & $\begin{array}{c}\text { Standard } \\
\text { error }\end{array}$ & \multicolumn{2}{|c|}{$95 \%$} \\
\cline { 3 - 5 } & & & $\begin{array}{c}\text { Lower } \\
\text { bound }\end{array}$ & $\begin{array}{c}\text { Upper } \\
\text { bound }\end{array}$ \\
\hline \multicolumn{1}{c|}{$\alpha$} & 0.001 & 0.001 & -0.001 & 0.004 \\
\hline$\beta$ & 2.498 & 0.290 & 1.922 & 3.073 \\
\hline$\gamma$ & 0.481 & 0.058 & 0.366 & 0.596 \\
\hline
\end{tabular}

The general expression of the model, therefore, assumes the following shape:

$$
a_{n}(t)=0.001 \frac{V_{n}\left(t-\tau_{n}\right)^{2.498}}{\Delta X_{n}\left(t-\tau_{n}\right)^{0.481}} \cdot \Delta V_{n}^{\text {front }}\left(t-\tau_{n}\right)+\varepsilon_{n}(t)
$$

The calibration of the model displays comforting results. The estimated parameters are all correct in sign and, therefore, highlight that the proportionality of the variables is as expected. Moreover, the order of the estimates corresponds to that obtained over the years by previous studies (table 2). The only differences can be ascribed to the different calibration and testing context of the model. In fact, almost all the models of this type in the literature emerge out of data gathering from fast stretches of road, having profoundly different flow characteristics from roundabouts. Regarding the remaining random variables $\left(\varepsilon_{\mathrm{n}}(\mathrm{t})\right)$, it was observed that they were normally with a mean equal to -0.047 and standard deviation equal to 0.237 .

Table 2: $\quad$ Estimated parameters of the GMN model by literature.

\begin{tabular}{|c|c|c|c|}
\hline & $\alpha$ & $\beta$ & $\gamma$ \\
\hline May and Keller [7] & $1.33 \times 10^{-4}$ & 0.80 & 2.80 \\
\hline Aron [10] & 2.45 & 0.66 & 0.70 \\
\hline Ozaki [32] & 1.10 & 0.90 & 1.00 \\
\hline Subramanian [11] & 15.24 & 1.09 & 1.66 \\
\hline Ahmed [12] & $-4.18 \times 10^{-2}$ & - & 0.151 \\
\hline
\end{tabular}




\section{Conclusions}

The calibrated behavioural model provides important indications about the flow modality in car-following conditions in correspondence to roundabout intersections. Specifically, this model permits estimation of the user reaction to stimuli on their route at the intersection. This reaction is proportional to the sensitivity of the user. In this way, the effects of bad roundabout planning on users can be foreseen, despite the extreme variability that characterises the phenomenon. This result allows the optimisation of work on the geometry of a roundabout in order to increase user safety. The next step will be to study the application of the behavioural model in different contexts in order to make the study applicable in general. Only in this way can the variables be identified for any driver behaviour in any context.

\section{References}

[1] Guido G. and Vitale A., A behavioural lane changing model for roundabout design, Transaction on the Built Environment, Vol. 79, WIT Press, Southampton, UK, pp. 567-575 (ISSN 1746-4498; ISBN 1-84564019-5), 2005.

[2] Chandler R., Herman R., and Montroll E., Traffic dynamics; studies in car following, Operations Research 6, 165+, 1958.

[3] Gazis D., Herman R. and Potts B., Car-following theory of steady state traffic flow, Operations Research 9, 449+, 1959.

[4] Herman R., Montroll E. W., Potts B. and Rothery R. W., Traffic dynamics: analysis of stability in car following, Operations Research, 7, pp. 86-106, 1959.

[5] Montroll E.W., Acceleration and clustering tendency of vehicular traffic, in Proceedings of the Symposium on Theory of Traffic Flow, Research Laboratories, General Motors, pp. 147-157, New York: Elsevier, 1959.

[6] Gazis D., Herman R. and Rothery R., Nonlinear follow the leader models of traffic flow, Operations Research 9, 545+, 1961.

[7] May A. and Keller H., Non integer car following models, Highway Research Record 199, 1967.

[8] Heyes M. P., Ashworth R., Further research on car following models, Transportation Research 6, pp. 287-291, 1972.

[9] Ceder A., May A. D., Further evaluation of single and two regime traffic flow models, Transportation Research Record, 567, pp. 1-30, 1976.

[10] Aron M., Car following in an urban network: simulation and experiments, in Proceedings of Seminar D, $16^{\text {th }}$ PTRC Meeting, pp. 27-39, 1988.

[11] Subramanian H., Estimation of car-following models, Master's thesis, MIT, Department of Civil and Environmental Engineering, Cambridge, Massachusetts, 1996.

[12] Ahmed K. I., Modeling driver's acceleration and lane changing behaviour, Doctoral thesis in Transportation Systems and Decision Sciences, MIT, 
Department of Civil and Environmental Engineering, Cambridge, Massachusetts, 1999.

[13] Gipps P. G., A behavioural car-following model for computer simulation, Transportation Research B 15B, pp. 105-111, 1981.

[14] McDonald M., Brackstone M. and Jeffery D., Simulation of lane usage characteristics on 3 lane motorways, in Proceedings of the 27th ISATA Conference, Aachen, Germany, 1994.

[15] Kumamoto H., Nishi K., Tenmoku K. and Shimoura H., Rule based cognitive animation simulator for current lane and lane change drivers, in Proceedings of the Second World Congress on ATT, pp. 1746-1752, Yokohama, Japan, 1995.

[16] Helly, W., Simulation of Bottlenecks in Single Lane Traffic Flow, in Proceedings of the Symposium on Theory of Traffic Flow., Research Laboratories, General Motors, pp. 207-238, New York: Elsevier, 1959.

[17] Hanken A. and Rockwell T.H., A model of car following derived empirically by piece-wise regression analysis, in Proceedings of the 3rd International Symposium on the Theory of Traffic Flow, pp. 40-41, New York: Elsevier, 1967.

[18] Bekey G. A, Burnham G. O. and Seo J., Control theoretic models of human drivers in car following, Human Factors, 19 (4), pp. 399-413, 1977.

[19] Xing, J., A parameter identification of a car following model, in Proceedings of the Second World Congress on ATT, Yokohama, pp. 1739-1745, 1995.

[20] Michaels R. M., Perceptual factors in car following, in Proceedings of the Second International Symposium on the Theory of Road Traffic Flow, pp. 44-59, Paris: OECD, 1963.

[21] Evans L. and Rothery R., Perceptual thresholds in car following: a recent comparison, Transportation Science, 11 (1), 60-72., 1977.

[22] Wiedemann R. and Reiter U., Microscopic traffic simulation: the simulation system MISSION, background and actual state, CEC Project ICARUS (V1052), Final Report, vol. 2, Appendix A, Brussels: CEC, 1992.

[23] Cameron D., Proceedings of the 28th ISATA Conference, Advanced Transportation Systems Symposium, pp.475-484, Stuttgart, Germany, 1995.

[24] Kikuchi C. and Chakroborty P., Car following model based on a fuzzy inference system, Transportation Research Record, 1365, pp. 82-91, 1992.

[25] Rekersbrink A., Mikroskopische verkehrssimulation mit hilfe der fuzzy logic, Strass enverkehrstechnik, 2/95, 68-74, 1995.

[26] Brackstone M., McDonald M. and Wu J., Development of a fuzzy logic based microscopic motorway simulation model, in Proceedings of the IEEE Conference on Intelligent Transportation Systems (ITSC97), Boston, USA, 1997. 
[27] Mar J., Lin F., Lin H. and Hsu L., The car following collision prevention controller based on the fuzzy basis function network, Fuzzy Sets and Systems 139, pp. 167-183, 2003.

[28] Daganzo C.F., in Traffic Flow, Cellular Automata = Kinematic Waves, Research Report, UCB-ITS-RR-2004-5, ISSN 0192 4095, 2004.

[29] Zhao X. and Gao, Z., A control method for congested traffic induced by bottlenecks in the coupled map car-following model, Article in press, Physica A, Elsevier.

[30] Ge H.X., Dai S.Q. and Dong, L.Y., An extended car-following model based on intelligent transportation system application, Article in press. Physica A., Elsevier.

[31] Aycin M. F. and Benekohal R., A linear acceleration car following model development and validation, Transportation Research Board, 77th Annual Meeting, 1998.

[32] Ozaki H., Reaction and anticipation in the car following behaviour, Proceedings of the thirteenth international symposium on traffic and transportation theory, pp. 349-366, Berkeley, CA, USA, 1993. 